\title{
SCALING LIMITS FOR WIDTH TWO PARTIALLY ORDERED SETS: THE INCOMPARABILITY WINDOW
}

\author{
NAYANTARA BHATNAGAR* $*$ NICK CRAWFORD ${ }^{\dagger}$, ELCHANAN MOSSEL $^{\ddagger}$, AND ARNAB SEN $^{\S}$
}

\begin{abstract}
We study the structure of a uniformly randomly chosen partial order of width 2 on $n$ elements. We analyze the local structure by studying the number of elements incomparable to a random element in the poset. We show that under the appropriate scaling, the number of incomparable elements converges to the height of a one dimensional Brownian excursion at a uniformly chosen random time in the interval $[0,1]$, which follows the Rayleigh distribution.
\end{abstract}

\section{INTRODUCTION}

In this paper we study the typical properties of a model of random fixed width posets, first studied by Brightwell and Goodall in [10]. Recall that an antichain of a poset $P$ is a subset of mutually incomparable elements. The width of a poset is defined to be the size of the largest antichain in it.

The width is a natural measure of the complexity of the set of partial orders. Bounded width posets are of interest in algorithms $[14,11]$ and applications in artificial intelligence $[22,23]$ since they can be specified in a compact manner [11]. For example, to specify a width-1 order, it is enough to give for each element the minimal element larger than it. In fact, asymptotically, among the set of all partial orders on $[n]=\{1, \cdots, n\}$, relatively few have low width $[10,19]$.

1.1 Results. Let $\mathcal{P}_{n, k}$ denote the set of labeled posets on vertex set $[n]$ of width $k$. In [10], the size of $\mathcal{P}_{n, k}$ is estimated asymptotically for fixed $k$ up to factors polynomial in $n$. When $k=2$, the estimates for $\left|P_{n, 2}\right|$ are tight up to lower order terms. More precisely,

$$
\left|\mathcal{P}_{n, 2}\right|=\frac{(2 n+1) !}{(n+2) !}\left(\frac{4}{25}+O\left(n^{-1 / 2} \log ^{2} n\right)\right)
$$

\footnotetext{
* Department of Statistics, UC Berkeley, email:nayan@stat.berkeley.edu, Supported by DOD ONR grant N0014-07-1-05-06 and DMS 0528488.

$\dagger$ Department of Statistics, UC Berkeley, email:crawford@stat.berkeley.edu, Supported by DMS 0548249 (CAREER).

$\ddagger$ Department of Statistics and Department of Computer Science , UC Berkeley, email:mossel@stat.berkeley.edu, Supported by DOD ONR grant N0014-07-1-05-06, DMS 0528488, DMS 0548249 (CAREER), and a Sloan Fellowship.

$\S$ Department of Statistics, UC Berkeley, email:arnab@stat.berkeley.edu, Supported by DOD ONR grant N0014-07-1-05-06, DMS 0528488, and DMS 0548249 (CAREER).
} 
In this work, we continue the study of bounded width posets and analyze the local structure of random posets of width 2 . In particular, we consider the asymptotic distribution of the number of incomparable elements from the point of view of a random element of the poset. This is in line with the study of random combinatorial objects from the point of view of a random element, as is commonly done for random graphs $[5,17]$.

To any poset $P$ on $[n]$ one can associate a graph $G_{P}=(V, E)$ with vertices $V=[n]$ and edge set $E$ consisting of those pairs $\{i, j\} \subset[n]$ which are not comparable. The graph $G_{P}$ is called the incomparability graph of $P$. The incomparability graph $G_{P}$ captures much of the structure of the poset $P$. For example, the width is the size of the maximum clique of $G_{P}$ while the size of a maximum independent set is the size of the largest chain, where a chain is a subset of elements of the poset each pair of which is comparable. Let $I_{P}(i)$ be the degree in $G_{P}$ of an element $i \in[n]$. Thus $I_{P}(i)$ is exactly the number of elements incomparable to $i$ in $P$. We will also refer to $I_{P}(i)$ as the incomparability window of $i$.

Let $\mu$ be the uniform distribution on $\mathcal{P}_{n, 2}$, let $P$ be drawn according to $\mu$ and let $\mathcal{X}$ be a distribution that is uniform on $[n]$ and independent of $\mathcal{P}_{n, 2}$ and $\mu$. We show that in the limit as $n$ tends to infinity, $I_{P}(\mathcal{X})$ when scaled appropriately converges in distribution to the height of a Brownian excursion at a random time. We state the result more formally below.

Let $\left(F_{i}\right)_{i=1}^{\infty}$ be a sequence of cumulative distribution functions corresponding to random variables $\left(X_{i}\right)_{i=1}^{\infty}$ and let $F$ be distribution function corresponding to a random variable $X$. Recall (see for e.g. [13]) that the sequence $\left(X_{i}\right)_{i=1}^{\infty}$ is said to converge in distribution to $X$ if for every $a \in \mathbb{R}$ at which $F$ is continuous

$$
\lim _{n \rightarrow \infty} F_{n}(a)=F(a)
$$

and denote it by

$$
X_{n} \stackrel{D}{\Rightarrow} X
$$

Let $B^{\operatorname{ex}}(t), 0 \leq t \leq 1$ denote the Brownian excursion process on [0,1] (see e.g. [16]). Informally, a Brownian excursion is obtained from the standard Brownian motion $B(t)$ on $[0,1]$ by conditioning on the events $B(0)=B(1)=0$ and $B(t)>0$ for all $t \in(0,1)$. Let $U$ denote a uniform random variable on $[0,1]$ independent of $B^{\operatorname{ex}}(t)$. Our main results are as follows.

Theorem 1.1 Let $P$ be a poset drawn from the distribution $\mu$. With the notation defined above,

$$
\frac{1}{\sqrt{n}} I_{P}(\mathcal{X}) \stackrel{D}{\Rightarrow} \frac{1}{\sqrt{2}} B^{e x}(U)
$$

Remark 1. It was observed by D. Aldous in [1] that $2 B^{\mathrm{ex}}(U)$ is distributed as Rayleigh distribution with density $f(s)=s e^{-s^{2} / 2}, s>0$.

The height of a poset is the size of the longest chain in it. Let $h(P)$ denote the height of the poset $P$. 
Theorem 1.2 Let $P$ be a poset drawn from the uniform distribution $\mu$. Then

$$
\frac{h(P)-n / 2}{\sqrt{n}} \stackrel{D}{\Rightarrow}|Z|
$$

where $Z \sim N(0,1 / 16)$.

1.2 Definitions and Notation. Since we focus on fixed width posets with $k=2$, to simplify notation henceforth we drop the subscripts and let $\mathcal{P}$ denote the set of width 2 posets on $[n]$. A poset $P=([n],<)$ on ground set $[n]=\{1, \cdots, n\}$ will be called a labeled poset. The set $\mathcal{P}$ is thus the set of labeled width-2 posets. Let $\langle P\rangle$ be the set of posets isomorphic to $P$. Such a class $\langle P\rangle$ is called an unlabeled poset.

By Dilworth's theorem (see e.g. [4]), the ground set $X$ of a width-2 poset $P=(X,<)$ can be partitioned into two chains, possibly in more than one way. Let $P=(L \cup R,<)$ be a poset where $L \cup R$ is a disjoint union of $[n]$ and $L$ and $R$ are chains in $P$. Then $(L, R,<)$ is called a two chain cover (of $P)$.

Let $T_{1}=(A, B,<)$ and $T_{2}=\left(A^{\prime}, B^{\prime},<\right)$ be two-chain covers (of possibly different labeled partial orders). Then $T_{1}$ and $T_{2}$ are isomorphic iff $|A|=\left|A^{\prime}\right|$ and there is a bijection taking each element of $A$ to an element of $A^{\prime}$ and each element of $B$ to an element of $B^{\prime}$ which preserves the relations of the partial order. An isomorphism class of two-chain covers consists of those two chain covers which are isomorphic to a specific two-chain cover. Such an isomorphism class is called an unlabeled two-chain cover and the set of all unlabeled two-chain covers of posets in $\mathcal{P}$ will be denoted by $\mathcal{T}$. Note that isomorphic posets have the same sets of unlabeled two chain covers.

The proof of Theorem 1.1 is based on the construction of a bijection between a set of unlabeled two-chain covers and constrained pairs of non-hitting random walks on $\mathbb{Z}$. We then reduce the analysis of the local structure of the two-chain covers to the analysis of the asymptotic properties of these walks. This refines the approach of [10] which used a bijection between $\mathcal{T}$ and a single constrained random walk on the lattice to enumerate the size of $\mathcal{P}$.

1.3 Related Models of Random Posets. The study of the asymptotic enumeration of posets was initiated by Kleitman and Rothschild in [19], where it was shown that almost all posets have height 3 and width approximately $n / 2$.

There are a number of other models of random posets (see for example the survey by Brightwell [9]). For example, one can fix the fraction $0<\alpha<\frac{1}{2}$ of comparable pairs and choose the poset uniformly from among those posets with $\left\lfloor\alpha n^{2}\right\rfloor$ comparable pairs [12]. The asymptotic analysis of the evolution of the number of posets as a function of $\alpha$ is given in $[20,21]$. In a second model, one takes $d$ linear orders on $[n]$ chosen uniformly at random and intersects them to produce a poset [24,7]. The distribution of the heights of these posets was analyzed in [6] and [2].

1.4 Open Problems. In our opinion, an interesting open question is to obtain results analogous to Theorem 1.1 for the distribution of $I_{P}(i)$ for width- $k$ posets when $k>2$. Unlike the width-2 case, it is not immediately clear how the uniform measure on labeled 
width- $k$ posets gets transferred to the geometric construction. Other open questions are stated in Section 6.

1.5 Organization of the Paper. The remainder of this paper is organized as follows. In Section 2 we show that the asymptotic analysis for width-2 posets can be reduced to the analysis of width-2 posets with one factor (a factor of a poset will be defined later). In Section 3 we construct a bijection between two chain covers with one factor and pairs of non-hitting random walks on the lattice and relate statistics of the random walks to the sizes of incomparability windows. In Section 4, we give technical estimates on the asymptotics of the walks which allow us to compute the limiting distribution of the incomparability window. Finally, we find the limiting distribution for the heights of width-2 posets in Section 5.

\section{Reduction to Posets with One Factor}

In this section we reduce the problem of determining the limiting distribution of the scaled incomparability window of a random element for a random poset to the corresponding problem for posets with exactly one factor (see definition below). This reduction simplifies much of the analysis in the subsequent sections.

Definition 2.1 ([10]) Let $P \in \mathcal{P}$ be a labeled width-2 poset and let $A_{1}, \cdots, A_{k}$ be the vertex sets of the connected components of the incomparability graph $G_{P}$. Further, let $P_{i}$ be the restriction of $P$ to $A_{i}$. The poset $P_{i}$ is called a factor of $P$.

It can be verified that the factors of a poset can be ordered so that $P_{1}<\cdots<P_{k}$, where $P_{i}<P_{j}$ means all elements of $P_{i}$ are less than all elements of $P_{j}$.

Let $\mathcal{P}_{0} \subseteq \mathcal{P}$ be the set of posets with one factor and let $\mu_{0}$ be the uniform distribution over $\mathcal{P}_{0}$.

Lemma 2.2 Let $Q$ be a poset chosen uniformly at random according to $\mu_{0}$ and let $\mathcal{X}$ be uniform over the ground set $[n]$ independent of $Q$. Let $B^{\mathrm{ex}}$ be the standard Brownian Excursion on $[0,1]$ and $U \sim$ Uniform $[0,1]$ independent of $B^{\mathrm{ex}}$. Suppose that

$$
\frac{I_{Q}(\mathcal{X})}{\sqrt{n}} \stackrel{D}{\Rightarrow} \frac{1}{\sqrt{2}} B^{\operatorname{ex}}(U)
$$

Then, if $P$ is a poset chosen according to $\mu$ and $\mathcal{X}$ is uniform over the ground set $[n]$ and independent of $P$

$$
\frac{I_{P}(\mathcal{X})}{\sqrt{n}} \stackrel{D}{\Rightarrow} \frac{1}{\sqrt{2}} B^{\operatorname{ex}}(U)
$$

Theorem 1.1 is then reduced to proving the hypothesis (2.1), and will be proved in Section 4. The proof of Lemma 2.2 relies on the following result of Brightwell and Goodall (see page 329 of [10]) showing that asymptotically, most width-2 posets have one large factor. 
Lemma 2.3 ([10]) Let $P$ be chosen uniformly at random from $\mathcal{P}$. Then

$$
\mathbb{P}\left(G_{P} \text { has a factor of size } \geq n-\log n\right) \rightarrow 1
$$

as $n \rightarrow \infty$.

Proof of Lemma 2.2. Let $\mathcal{X}$ be chosen uniformly from the ground set $[n]$. Consider the random variable $I_{P}(\mathcal{X})$ and let $M$ denote the largest connected component of $G_{P}$. By Lemma 2.3 , for $0<a<b$,

$$
\mathbb{P}\left(I_{P}(\mathcal{X}) \in(a \sqrt{n}, b \sqrt{n}]\right)=\mathbb{P}\left(I_{P}(\mathcal{X}) \in(a \sqrt{n}, b \sqrt{n}] \cap\{|M| \geq n-\log n\}\right)+o(1) .
$$

Conditioning on the size of $M$, we have that $\mathbb{P}(\mathcal{X} \in M|| M \mid)=n^{-1}|M|$, so we may further write

$$
\mathbb{P}\left(I_{P}(\mathcal{X}) \in(a \sqrt{n}, b \sqrt{n}]\right)=\mathbb{P}\left(I_{P}(\mathcal{X}) \in(a \sqrt{n}, b \sqrt{n}|| \mathcal{X} \in M,|M| \geq n-\log n)+o(1) .\right.
$$

For each $m \geq n-\log n$, let $F_{m}=\{\mathcal{X} \in M\} \cap\{|M|=m\}$. Since with high probability $\mathcal{X}$ is chosen from $M$ when $|M| \geq n-\log n$,

$$
\mathbb{P}\left(I_{P}(\mathcal{X}) \in(a \sqrt{n}, b \sqrt{n}]\right)=\sum_{m \geq n-\log n} \mathbb{P}\left(I_{P}(\mathcal{X}) \in(a \sqrt{n}, b \sqrt{n}] \mid F_{m}\right) \mathbb{P}\left(F_{m}\right)+o(1) .
$$

By construction, the distribution of $I_{P}(\mathcal{X})$ conditioned on $F_{m}$ is the same as the distribution of $I_{Q}(\mathcal{Y})$ where $Q$ is a random poset with one factor on the ground set $[m]$ and $\mathcal{Y}$ is uniform on $[m]$. In other words,

$$
\mathbb{P}\left(I_{P}(\mathcal{X}) \in(a \sqrt{m}, b \sqrt{m}] \mid F_{m}\right)=\mathbb{P}\left(I_{Q}(\mathcal{Y}) \in(a \sqrt{m}, b \sqrt{m}]\right) .
$$

Thus from (2.1), for all $\epsilon>0$, there is $m(\epsilon)$ so that for $m>m(\epsilon)$,

$$
\left|\mathbb{P}\left(I_{P}(\mathcal{X}) \in(a \sqrt{m}, b \sqrt{m}] \mid F_{m}\right)-\mathbb{P}\left(\frac{1}{\sqrt{2}} B^{\operatorname{ex}}(U) \in(a, b]\right)\right| \leq \epsilon .
$$

Choosing $n$ large enough so that $n-\log n \geq m(\epsilon)$ and $o(1) \leq \epsilon$ we have

$$
\left|\mathbb{P}\left(I_{P}(\mathcal{X}) \in(a \sqrt{n}, b \sqrt{n}]\right)-\mathbb{P}\left(\frac{1}{\sqrt{2}} B^{\operatorname{ex}}(U) \in(a, b]\right)\right| \leq \epsilon \sum_{m \geq n-\log n} \mathbb{P}\left(F_{m}\right)+o(1)
$$

$\leq 2 \epsilon$

and the claim follows.

As a consequence of Lemma 2.2, we can henceforth restrict ourselves to working over $\mathcal{P}_{0}$, the set of labeled posets with just a single factor.

\section{Combinatorial Mappings}

In this section we construct a correspondence between posets $\mathcal{P}_{0}$ and a random walk representation useful for our probabilistic analysis. We then relate certain statistics of the random walks to the size of the incomparability window. The idea behind the correspondence is essentially due to Brightwell and Goodall, but requires some modification in order to describe the incomparability window. 
Definition 3.1 (Two-chain cover [10]) Let $P=(L \cup R,<)$ be a poset where $L \cup R$ is a disjoint union of $[n]$ and $L$ and $R$ are chains in $P$. Then $(L, R,<)$ is called a two chain cover (of $P$ ).

Definition 3.2 (Unlabeled two-chain cover [10]) Let $T_{1}=(A, B,<)$ and $T_{2}=\left(A^{\prime}, B^{\prime},<\right)$ be two two-chain covers. Then $T_{1}$ and $T_{2}$ are isomorphic iff $|A|=\left|A^{\prime}\right|$ and there is a bijection taking each element of $A$ to an element of $A^{\prime}$ and each element of $B$ to an element of $B^{\prime}$ which is an isomorphism of the partial orders. An isomorphism class of two chain covers is called an unlabeled two-chain cover.

Denote the set of unlabeled two chain covers with one factor by $\mathcal{T}_{0}$. We say that $T_{u} \in \mathcal{T}_{0}$ is an unlabeled two-chain cover of a poset $P$ if there is $T^{\prime} \in T_{u}$ which is a two-chain cover of $P$.

For each unlabeled two chain cover $T_{u}$, let $T=(A, B,<)$ be the element of $T_{u}$ with $A=\{1, \cdots, k\}$ and $B=\{k+1, \cdots, n\}$ for $1 \leq k \leq n$ so that the chains $A$ and $B$ are ordered in the natural way. The two chain cover $T$ will be the canonical representative of $T_{u}$ and we will identify elements of $\mathcal{T}_{0}$ with their canonical representatives. By abuse of notation, by $T=(A, B,<) \in \mathcal{T}_{0}$ we will mean the element of $\mathcal{T}_{0}$ which is the isomorphism class of $T$.

The following correspondence between $\mathcal{P}_{0}$ and $\mathcal{T}_{0}$ was given in [10]. Let $P \in \mathcal{P}_{0}$ and define

$$
\psi(P)=\left\{T \in \mathcal{T}_{0}: T \text { is an unlabeled two-chain cover of } P\right\} .
$$

Note that $|\psi(P)| \leq 2$. It can be verified that $\psi$ associates disjoint sets of covers with non-isomorphic posets and associates the same set of covers with any two isomorphic posets. For any unlabeled two-chain cover $T$, define $\psi^{-1}(T)$ to be the set of posets $P$ in $\mathcal{P}_{0}$ such that $T$ is an unlabeled two chain cover of $P$. The random walk representation we study is as follows.

Definition 3.3 A pair of non-hitting walks $(V, W)$ of length $n$ on the integer lattice is defined to be a pair of walks where

(1) $V(0)=W(0)=0$.

(2) For every $0 \leq t<n,|V(t+1)-V(t)|=|W(t+1)-W(t)|=1$.

(3) $V(n)=W(n)$

(4) For every $1 \leq t<n, V(t)>W(t)$.

Let $\Gamma_{n}^{0}$ be the set of non-hitting pairs of walks of length $n$. For $(V, W) \in \Gamma_{n}^{0}$, define the height function $H_{V, W}:[n] \rightarrow\{0,2,4, \cdots\}$ by

$$
H_{V, W}(k):=V(k)-W(k) \quad 1 \leq k \leq n .
$$

Definition 3.4 Let $P=([n],<)$ be a labeled poset. Let us define the function $\tau_{P}:[n] \rightarrow$ $[n]$ by

$$
\tau_{P}(x)=|\{y \in[n]: y<x\}|+1
$$


The first result of this section relates the incomparability window $I_{P}(x)$ for an element $x$ of a poset $P \in \mathcal{P}_{0}$ to $H_{V, W}\left(\tau_{P}(x)\right)$ for walks $(V, W) \in \Gamma_{n}^{0}$ associated with $P$.

The set of width-2 posets and non-hitting walks are associated as follows. We will construct a bijection $\gamma$ between $\mathcal{T}_{0}$ and the set of non-hitting walks $\Gamma_{n}^{0}$ in Section 3.1. Given $\gamma$, let

$$
\phi(P)=\gamma(\psi(P))
$$

to be the image of $\psi(P)$ under $\gamma$. The incomparability window can then be related to the height function $H_{V, W}$ as follows.

Theorem 3.5 Let $P \in \mathcal{P}_{0},(V, W) \in \phi(P)$ and let $x \in[n]$ be an element of the ground set. Then,

$$
\left|I_{P}(x)-H_{V, W}\left(\tau_{P}(x)\right)\right| \leq \operatorname{Err}(P, x)
$$

where

$$
\operatorname{Err}(P, x):=\left|V\left(\tau_{P}(x)+I_{P}(x)\right)-V\left(\tau_{P}(x)\right)\right|+\left|W\left(\tau_{P}(x)+I_{P}(x)\right)-W\left(\tau_{P}(x)\right)\right| .
$$

At a high level, the argument for why the above bound is useful is as follows. We will show that under $\phi$ the uniform distribution on $\mathcal{P}_{0}$ induces the uniform distribution over $\Gamma_{n}^{0}$. For $(V, W)$ chosen uniformly from $\Gamma_{n}^{0}$, we expect that under diffusive scaling, $H_{V, W}$ converges to a Brownian excursion. Our goal is to show that for a uniformly random $P$ and a uniformly random element $\mathcal{X}$, the scaled error $\operatorname{Err}(P, \mathcal{X}) / \sqrt{n}$ goes to 0 in probability. In Section 4 we show that with high probability the size of the incomparability window is bounded by $n^{2 / 3}$. Therefore, bounding the scaled error reduces to bounding the maximum fluctuation of each the walks $V$ and $W$ in $n^{2 / 3}$ steps. We will show that for random $P$ and $x$, the differences $\left|V\left(\tau_{P}(x)+I_{P}(x)\right)-V\left(\tau_{P}(x)\right)\right|$ are at most $n^{1 / 3}$ with high probability and hence the scaled error goes to 0 .

Define the joint distribution $\omega$ on $\mathcal{P}_{0} \times \mathcal{T}_{0} \times \Gamma_{n}^{0}$ as follows. Let $P \sim \mu_{0}$ be chosen uniformly from $\mathcal{P}_{0}$. Given $P$, choose $T$ uniformly from $\psi(P)$. Given $P$ and $T$, let $(V, W)=\gamma(T)$ with probability 1 . Let $\rho_{0}$ and $\sigma_{0}$ be the marginals of $T$ and $(V, W)$, i.e. for every $T \in \mathcal{T}_{0}$

$$
\rho_{0}(T)=\sum_{P: T \in \psi(P)} \frac{\mu_{0}(P)}{|\psi(P)|}
$$

and $(V, W) \in \Gamma_{n}^{0}$

$$
\sigma_{0}(V, W)=\sum_{P:(V, W) \in \phi(P)} \frac{\mu_{0}(P)}{|\phi(P)|} .
$$

Lemma 3.6 ([10]) Let $\rho_{0}$ be defined as above. The distribution $\rho_{0}$ is uniform over $\mathcal{T}_{0}$.

Lemma 3.7 Let $\sigma_{0}$ be defined as above. Then $\sigma_{0}$ is the uniform distribution over $\Gamma_{n}^{0}$.

The proof is postponed until after the construction of the bijection $\gamma$ (Proposition $3.13)$. 
Definition 3.8 Let text deleted $(V, W)=\gamma(T)$. For $x \in[n]$, define the function $\tau_{V, W}(x)=1+\left|\left\{y \quad: \quad y<_{T} x\right\}\right|$ where $y<_{T} x$ denotes that $y<x$ in the partial order defined by $T$.

Lemma 3.9 Let $(P,(V, W))$ be chosen according to the two-dimensional marginal of $\omega$ on $\mathcal{P}_{0} \times \Gamma_{n}^{0}$. Let $\mathcal{X}$ be a uniform element of the ground set $[n]$ independent of $(P,(V, W))$. Then, there exists a random variable $\mathcal{C}$ on the product probability space which is uniform over $[n]$ and independent of $(V, W)$ such that

$$
H_{V, W}\left(\tau_{P}(\mathcal{X})\right) \equiv H_{V, W}\left(\tau_{V, W}(\mathcal{C})\right)
$$

Proof. Since $P$ and $(V, W)$ are chosen according to the two-dimensional marginal of $\omega$, $(V, W) \in \phi(P)$. Let $T=\gamma^{-1}((V, W))$. In particular $T \in \psi(P)$ where we are using the convention that $T$ is the canonical representative of an unlabeled two-chain cover $T_{u}$ of $P$. Since $T \in \psi(P)$, for any (labeled) two-chain cover $T_{P} \in T_{u}$ of $P$ there is a (unique) two-chain isomorphism $\alpha$ between $T_{P}$ and $T$. Consequently,

$$
\begin{aligned}
\tau_{P}(x) & =1+|\{y \in[n]: y<x\}| \\
& =1+\left|\left\{y \in[n]: y<_{T_{P}} x\right\}\right| \\
& =1+\left|\left\{y \in[n]: y<_{T} \alpha(x)\right\}\right|=\tau_{V, W}(\alpha(x)) .
\end{aligned}
$$

Given $P$ and $(V, W) \in \phi(P)$. Let

$$
\mathcal{C}=\alpha(\mathcal{X})
$$

Note that though the map $\alpha$ depends on $P,(V, W)$ and the choice of $T_{P}$, since $\mathcal{X}$ is uniform over $[n]$ and independent of everything else, $\mathcal{C}$ is also uniform over $[n]$ and independent of $P$ and $(V, W)$. The assertion of the lemma follows.

In order to formalize the high level argument above we would like to relate the distribution of $H_{V, W}\left(\tau_{V, W}(\mathcal{C})\right)$ for a uniformly random element $\mathcal{C}$ to the distribution of $H_{V, W}$ evaluated at a uniformly random time. This is the main result of the section.

Theorem 3.10 (Symmetrization) Let $(V, W)$ be chosen at random from $\Gamma_{n}^{0}$ according to $\sigma_{0}$, and let $T=\gamma^{-1}(V, W)=(A, B,<)$. Let $\mathcal{C}$ be a uniform element from $[n]$ independent of $(V, W)$ and let $U_{n}$ be uniform on $\{1,2, \ldots, n\}$ independent of $(V, W)$. Then for every $m \in 2 \mathbb{Z}_{\geq}$

$$
\mathbb{P}\left(H_{V, W}\left(\tau_{V, W}(\mathcal{C})\right)=m\right)=\mathbb{P}\left(H_{V, W}\left(U_{n}\right)=m\right) .
$$

3.1 The Bijection $\gamma$ from Two-chain Covers to Non-hitting Walks. Next, we construct a bijection $\gamma$ from $\mathcal{T}_{0}$ to the set of pairs of walks $\Gamma_{n}^{0}$. Our construction deviates from that of [10] since we construct a bijection to pairs of walks rather than a single walk.

Given a two chain cover $T=(A, B,<)$ in $\mathcal{T}_{0}$, let $(\lambda, \delta)$ denote the pair of total orders, defined by adding to the poset the following relations: if $a \in A, b \in B$ are not comparable in $T$, then we set $a<b$ in $\lambda$ and set $a>b$ in $\delta$. Following the terminology of [10], we call $(\lambda, \delta)$ the greedy pair of orders associated with $T$. The relations of the two chain cover can be reconstructed by setting $a<b$ (resp. $>$ ) if $a$ precedes (resp. follows) $b$ in both of the greedy total orders. 
For $c \in[n]$, denote by $\lambda(c)$ the number of elements less than or equal to $c$ in $\lambda$ and define $\delta(c)$ similarly. For $1 \leq i \leq n$, let $\lambda_{i}$ (resp. $\delta_{i}$ ) denote the element in position $i$ in $\lambda(\operatorname{resp} . \delta)$.

Proposition $3.11([10])$ A pair of orders $(\lambda, \delta)$ on $[n]$ is a greedy pair for some twochain cover $T=(A, B,<)$ iff for every $a \in A$ and $b \in B$, if $\delta(a)<\delta(b)$ then $\lambda(a)<\lambda(b)$.

Given the pair of greedy orders $(\lambda, \delta)$ obtained from $T$, we define a pair of walks $(V, W)$ as follows: Start by setting $V(0)=W(0)=0$. In the $i$-th step, the walk $V$ (resp. $W$ ) takes a step up if the $i$-th element of the total order $\lambda$ (resp. $\delta$ ) belongs to $A$, and otherwise it takes a step down. In other words

$$
V(i)=\left\{\begin{array}{ll}
V(i-1)+1 & \text { if } \lambda_{i} \in A \\
V(i-1)-1 & \text { if } \lambda_{i} \in B
\end{array} \quad W(i)= \begin{cases}W(i-1)+1 & \text { if } \delta_{i} \in A \\
W(i-1)-1 & \text { if } \delta_{i} \in B .\end{cases}\right.
$$

For $1 \leq k \leq n$, let $\pi_{k}(V):=V(k)-V(k-1) \in\{-1,1\}$ denote the $k$-th step of $V$ and define $\pi_{k}(W)$ analogously.

Proposition 3.12 Let $T \in \mathcal{T}_{0}$. Then $\gamma(T)=(V, W)$ is a pair of non-hitting walks.

Proof. Recall that non-hitting walks satisfy four properties (see Definition 3.3). Properties (1), (2) and (3) follow immediately by the definition of $\gamma$. Suppose that (4) does not hold, so that in particular, there is a smallest $k, 0<k<n$ such that $V(k)=W(k)$. The claim is that then $T$ must have more than one factor, a contradiction.

By the definition of $\lambda$ and $\delta$, every element $a \in A$ satisfies $\lambda(a) \leq \delta(a)$ and conversely, every element of $b \in B$ satisfies $\delta(b) \leq \lambda(b)$. Since $V(k)=W(k)$, by construction the sets $S_{\lambda}=\left\{\lambda_{1}, \cdots, \lambda_{k}\right\}$ and $S_{\delta}=\left\{\delta_{1}, \cdots, \delta_{k}\right\}$ consist of the same numbers of elements from $A$ and $B$. By considering any $\lambda_{i} \in B \cap S_{\lambda}$, it must be that $\lambda_{i} \in S_{\delta}$. A symmetric argument for $\delta_{j} \in A \cap S_{\delta}$ implies $\delta_{j} \in S_{\lambda}$, and hence, $S_{\lambda}=S_{\delta}=S$.

Since $|S|=k<n$, there exists an element $x \notin S$ appearing in both $\lambda$ and $\delta$ above the elements of $S$. The element $x$ must therefore be in a different component of the incomparablity graph of the poset $P$ defined by $T$ than the elements of $S$, implying that $T$ has more than one factor.

Proposition 3.13 The map $\gamma: \mathcal{T}_{0} \rightarrow \Gamma_{n}^{0}$ is a bijection.

Proof. By the definition of the map $\gamma$, distinct two chain covers are mapped to nonisomorphic greedy orders. Moreover, non-isomorphic greedy orders are mapped to distinct walks $(V, W)$ and by Proposition 3.12 the walks are non-hitting. Hence $\gamma$ is one-to-one. It remains to show that any pair of walks corresponds to some two chain cover.

From a pair of walks $V, W \in \Gamma_{n}^{0}$ define a pair of total orders $(\lambda, \delta)$ on $[n]$ and sets $A$ and $B$, where $A \cup B=[n]$ as follows. Let $A=\{1, \cdots, k\}$ and $B=\{k+1, \cdots, n\}$, where $k=$ $\left|\left\{t: \pi_{t}(V)=+1\right\}\right|=\left|\left\{t: \pi_{t}(W)=+1\right\}\right|$ and the equality follows since $V(n)=W(n)$. The greedy orders are defined as follows. Let $V_{+}=\left(i_{1}, \cdots, i_{|A|}\right)$ be the (increasing) subsequence of indices so that $\pi_{i_{\ell}}(V)=+1$. Analogously, let us define the subsequences of indices $V_{-}=\left(i_{|A|+1}, \cdots, i_{n}\right), W_{+}=\left(j_{1}, \cdots, j_{|A|}\right)$, and $W_{-}=\left(j_{|A|+1}, \cdots, j_{n}\right)$. The linear orders $\lambda$ and $\delta$ are then defined by the rankings $\lambda_{i_{\ell}}=\ell$ and $\delta_{j_{\ell}}=\ell$ for each $\ell \in[n]$. 
By property (4) of the walks $(V, W)$, there are no elements $a \in A$ and $b \in B$ such that $\delta(a)<\delta(b)$ but $\lambda(a)>\lambda(b)$. Therefore the pair $(\lambda, \delta)$ satisfies the condition of Proposition 3.11 and is a greedy pair of linear orders corresponding to some two-chain cover. Hence, the map $\gamma$ is a bijection.

Lemma 3.7 is now easy corollary.

Proof of Lemma 3.7 . Let $P$ be a width-2 poset in $\mathcal{P}_{0}$. Let $\psi$ and $\phi$ as defined in (3.1) and (3.3). Suppose that $\left(V_{1}, W_{1}\right)$ and $\left(V_{2}, W_{2}\right)$ are walks in $\Gamma_{n}^{0}$. Let $T_{1}=\gamma^{-1}\left(V_{1}, W_{1}\right)$ and $T_{2}=\gamma^{-1}\left(V_{2}, W_{2}\right)$. Then,

$$
\begin{aligned}
& \sigma_{0}\left(V_{1}, W_{1}\right)=\sum_{P \in \phi^{-1}\left(V_{1}, W_{1}\right)} \frac{\mu_{0}(P)}{|\phi(P)|}=\sum_{P \in \psi^{-1}\left(T_{1}\right)} \frac{\mu_{0}(P)}{|\psi(P)|} \\
= & \sum_{P \in \psi^{-1}\left(T_{2}\right)} \frac{\mu_{0}(P)}{|\psi(P)|}=\sum_{P \in \phi^{-1}\left(V_{2}, W_{2}\right)} \frac{\mu_{0}(P)}{|\phi(P)|}=\sigma_{0}\left(V_{2}, W_{2}\right)
\end{aligned}
$$

The third equality follows from Lemma 3.6 and the fourth by Proposition 3.13.

Definition 3.14 Let $T=(A, B,<) \in \mathcal{T}_{0}$. For $i \in[n]$, define the incomparability window of $i$ in $T, I_{T}(i):=I_{P}(i)$ where $P$ is the poset defined by $T$.

The elements of $T=(A, B,<) \in \mathcal{T}_{0}$ can be labeled $a_{1}<\cdots<a_{k}$ and $b_{1}<\cdots<b_{n-k}$. For each $1 \leq i \leq k$, let $t_{V}(i)$ be the time at that the walk $V$ has taken $i$ steps up and let $t_{W}(i)$ be the first time $W$ has taken $i$ steps up. Similarly, for each $1 \leq j \leq n-k$ let $s_{V}(j)$ and $s_{W}(j)$ be the first times that $V$ and $W$ have taken $j$ downward steps respectively.

Lemma 3.15 Let $n$ be fixed and let $T=(A, B,<)$ be a two chain cover in $\mathcal{T}_{0}$. Then we have the following identifications:

(1) The size of the incomparability window for $a_{i}$ satisfies

$$
I_{T}\left(a_{i}\right)=t_{W}(i)-t_{V}(i) .
$$

Similarly, for an element $b_{j}$,

$$
I_{T}\left(b_{j}\right)=s_{V}(j)-s_{W}(j)
$$

(2) Alternately,

$$
I_{T}\left(a_{i}\right)=\min \left\{k: k=H_{V, W}\left(t_{V}(i)\right)+W\left(t_{V}(i)\right)-W\left(t_{V}(i)+k\right)\right\}
$$

and

$$
I_{T}\left(b_{j}\right)=\min \left\{k: k=V\left(s_{W}(j)+k\right)-V\left(s_{W}(j)\right)+H_{V, W}\left(s_{W}(j)\right)\right\} .
$$

Proof. By symmetry we may restrict attention to the statements for $a_{i} \in A$. We first prove part (1). At time $t_{V}(i)$ when $V$ has taken $i$ steps 'up', the set $\left\{\lambda_{1}, \cdots, \lambda_{t_{V}(i)}\right\}$ consists of $a_{i}$ and all the elements below $a_{i}$. Similarly, when $W$ has taken $i$ steps 'up', at 

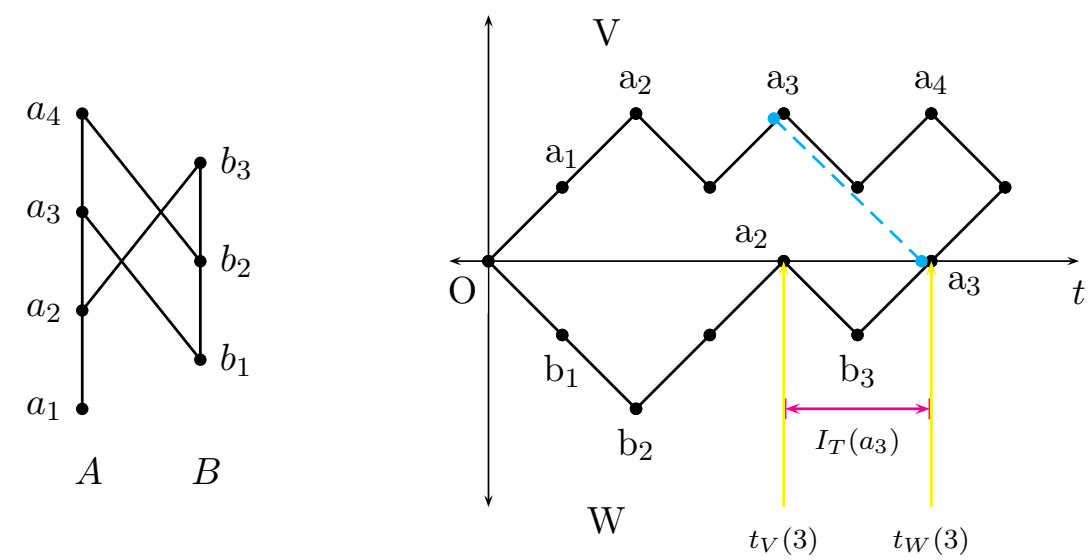

Figure 1: (Left) A two chain covering $(A, B,<)$ of a poset with 7 elements. (Right) The associated pair of non-crossing walks $(V, W)$. The incomparability window for the element $a_{3}$ is illustrated.

time $t_{W}(i)$, the set $\left\{\delta_{1}, \cdots, \delta_{t_{W}(i)}\right\}$ consists of $a_{i}$, the elements below $a_{i}$ or incomparable to it, and no elements that are above $a_{i}$. Hence, $t_{W}(i)-t_{V}(i)$ is exactly the number of elements that are incomparable to $a_{i}$ (see Figure 1).

Next we consider the second description of the incomparability window for $a_{i} \in A$. Let $r$ be the number of up steps in $W$ up to time $t_{V}(i)$. Then the locations of the walks $V$ and $W$ at time $t_{V}(i)$ are given by

$$
V\left(t_{V}(i)\right)=2 i-t_{V}(i) \text { and } W\left(t_{V}(i)\right)=2 r-t_{V}(i) .
$$

Therefore,

$$
V\left(t_{V}(i)\right)-W\left(t_{V}(i)\right)=2(i-r)
$$

By part $(\mathrm{a}), I_{T}\left(a_{i}\right)$ is the minimum steps required by $W$ to have $(i-b)$ more upward steps after time $t_{V}(i)$. So, we can write

$$
\begin{aligned}
I_{T}\left(a_{i}\right) & =\min \left\{k: W\left(t_{V}(i)+k\right)-W\left(t_{V}(i)\right)=2(i-r)-k\right\} \\
& =\min \left\{k: W\left(t_{V}(i)+k\right)-W\left(t_{V}(i)\right)=V\left(t_{V}(i)\right)-W\left(t_{V}(i)\right)-k\right\} \quad \text { by }(3.9) \\
& =\min \left\{k: k=V\left(t_{V}(i)\right)-W\left(t_{V}(i)+k\right)\right\} \\
& =\min \left\{k: k=H_{V, W}\left(t_{V}(i)\right)+W\left(t_{V}(i)\right)-W\left(t_{V}(i)+k\right)\right\} .
\end{aligned}
$$

Equation 3.8 follows by a symmetric argument.

Via linear interpolation, we may view $(V, W)$ as a pair of polygonal paths in $\mathbb{R}^{2}$. Let $A(T)$ denote the area between the two (piecewise linear) curves $V, W$. As a consequence of the preceding identification, we note that the sum of the incomparability windows can be written in terms of the area between the curves:

Corollary 3.16 $\sum_{c \in[n]} I_{T}(c)=A(T)$. 
Proof. We will show that $\sum_{i=1}^{k} I_{T}\left(a_{i}\right)=\frac{1}{2} A(T)=\sum_{i=1}^{n-k} I_{T}\left(b_{i}\right)$. The first equality follows by noticing that the lengths of the intervals $t_{W}(i)-t_{V}(i)$ are exactly the number of squares on the $i$-th diagonal (running from top left to bottom right) between $V$ and $W$ (see Figure 1). Since each square has a side length of $\sqrt{2}$, the area is 2 . The second equality follows by counting the squares between $V$ and $W$ from bottom left to top right.

Proof of Theorem 3.5. Fix a poset $P \in \mathcal{P}_{0}$ and let $(V, W) \in \phi(P)$. Let $T=\gamma^{-1}(V, W)=$ $(A, B,<)$ be the two-chain cover corresponding to $(V, W)$. Recall from (3.2) that for an element $x, \tau_{P}(x)$ is the number of elements of the poset smaller than or equal to $x$. Recall that we can think of the elements of $A$ being labeled $a_{1}, \cdots, a_{k}$ and those of $B$ being labeled $b_{1}, \cdots, b_{n-k}$. Let $T_{u}$ be the unlabeled two-chain cover of which $T$ is the canonical representative. Since $T \in \psi(P)$, for any labeled two-chain cover $T_{P}$ of $P$ in $T_{u}$, there is a two-chain isomorphism $\alpha$ between $T_{P}$ and $T$. Consequently, for any element $x \in[n]$,

$$
I_{P}(x)=I_{T_{P}}(x)=I_{T}(\alpha(x)) .
$$

Also, if $\alpha(x)=a_{i} \in A$ then $\tau_{P}(x)=t_{V}(i)$ while if $\alpha(x)=b_{j} \in B$, then $\tau_{P}(x)=s_{W}(j)$. Therefore, using the bounds (3.7) and (3.8) from Lemma 3.15, we have

$$
\begin{aligned}
\mid I_{P}(x) & -H_{V, W}\left(\tau_{P}(x)\right)|=| I_{T}(\alpha(x))-H_{V, W}\left(\tau_{P}(x)\right) \mid \\
& \leq\left|V\left(\tau_{P}(x)+I_{T}(\alpha(x))\right)-V\left(\tau_{P}(x)\right)\right|+\left|W\left(\tau_{P}(x)+I_{T}(\alpha(x))\right)-W\left(\tau_{P}(x)\right)\right| \\
& =\left|V\left(\tau_{P}(x)+I_{P}(x)\right)-V\left(\tau_{P}(x)\right)\right|+\left|W\left(\tau_{P}(x)+I_{P}(x)\right)-W\left(\tau_{P}(x)\right)\right| .
\end{aligned}
$$

Let $(V, W)$ be a random pair of non-hitting walks drawn from $\sigma_{0}$ and let $T=(A, B,<)$ be the corresponding unlabeled two chain cover. Let $\mathcal{C}$ be uniform over $[n]$. We show that $H_{V, W}\left(\tau_{V, W}(\mathcal{C})\right)$ is distributionally invariant if we replace $\tau_{V, W}(\mathcal{C})$ with an independent uniform variable $U_{n}$ on $\{1, \cdots, n\}$.

Proof of Theorem 3.10. Let $m \in 2 \mathbb{Z}_{\geq}$be fixed and define

$$
\mathcal{A}_{t}\left(e_{1}, e_{2}\right):=\left\{(V, W): H_{V, W}(t)=m,\left(\pi_{t}(V), \pi_{t}(W)\right)=\left(e_{1}, e_{2}\right)\right\}
$$

where $e_{i} \in\{-1,+1\}$. Define an involution $\imath: \Gamma_{n}^{0} \rightarrow \Gamma_{n}^{0}$ as follows:

$$
\imath(V, W):=\left(V^{\prime}, W^{\prime}\right) \text { where: }\left\{\begin{array}{l}
V^{\prime}(0)=W^{\prime}(0)=0, \\
\pi_{i}\left(V^{\prime}\right)=-\pi_{n-i+1}(V), \\
\pi_{i}\left(W^{\prime}\right)=-\pi_{n-i+1}(W)
\end{array}\right.
$$

for all $1 \leq i \leq n$. In words $\imath$ maps $(V, W)$ to its time reversal, additionally shifting the height so that $V(n), W(n)$ maps to $(0,0)$. It is easy to see that $\left(V^{\prime}, W^{\prime}\right) \in \Gamma_{n}^{0}$ and that $\imath^{2}=i d$. This implies that $\imath$ is a bijection on $\Gamma_{n}^{0}$ and thus preserves the uniform distribution $\sigma_{0}$ over $\Gamma_{n}^{0}$. 
We claim that

$$
\sum_{t=1}^{n}\left|\mathcal{A}_{t}(-1,+1)\right|=\sum_{t=1}^{n}\left|\mathcal{A}_{t}(+1,-1)\right| .
$$

This follows by observing that $\imath$ maps $\mathcal{A}_{t}(-1,+1)$ into $\mathcal{A}_{n-t+1}(+1,-1)$ and is a bijection. Indeed: $H_{\imath(V, W)}(n-t+1)=H_{V, W}(t)$ and if $\left(\pi_{k}(V), \pi_{k}(W)\right)=(-1,+1)$, then $\left(\pi_{n-t+1}\left(V^{\prime}\right), \pi_{n-t+1}\left(W^{\prime}\right)\right)=(+1,-1)$ as well.

Let $T=(A, B,<)$ be the two chain cover corresponding to $(V, W)$. Suppose for $c \in[n]$, $\tau_{V, W}(c)=t$. Then $\pi_{t}(V)=+1$ if and only if $c \in A$. Moreover there is exactly one element of $A$ such that $\tau_{V, W}(c)=t$. Similarly, $\pi_{t}(W)=-1$ if and only if $c \in B$, and there is exactly one such element $c$ in $B$. Therefore, for $1 \leq t \leq n$, we have

$$
\left|\left\{c \in[n]: \tau_{V, W}(c)=t\right\}\right|=\left\{\begin{array}{lll}
1 & \text { if } & \pi_{t}(V)=+1, \pi_{t}(W)=+1 \\
1 & \text { if } & \pi_{t}(V)=-1, \pi_{t}(W)=-1 \\
2 & \text { if } & \pi_{t}(V)=+1, \pi_{t}(W)=-1 \\
0 & \text { if } & \pi_{t}(V)=-1, \pi_{t}(W)=+1 .
\end{array}\right.
$$

Therefore, for every $m \in 2 \mathbb{Z}_{\geq}$,

$$
\begin{aligned}
& \mathbb{P}_{(V, W) \sim \sigma_{0}}\left(H_{V, W}\left(\tau_{V, W}(\mathcal{C})\right)=m\right) \\
& =\left|\Gamma_{n}^{0}\right|^{-1} n^{-1} \sum_{(V, W) \in \Gamma_{n}^{0}} \sum_{c \in[n]} \mathbb{I}\left(H_{V, W}\left(\tau_{V, W}(c)\right)=m\right) \\
& =\left|\Gamma_{n}^{0}\right|^{-1} n^{-1} \sum_{(V, W) \in \Gamma_{n}^{0}} \sum_{t=1}^{n}\left|\left\{c \in[n]: \tau_{V, W}(c)=t\right\}\right| \mathbb{I}\left(H_{V, W}(t)=m\right) .
\end{aligned}
$$

Switching the order of summation and using (3.12), we have

$$
\begin{aligned}
\mathbb{P}_{(V, W) \sim \sigma_{0}}\left(H_{V, W}\left(\tau_{V, W}(\mathcal{C})\right)=m\right) \\
=\left|\Gamma_{n}^{0}\right|^{-1} n^{-1} \sum_{t=1}^{n}\left|\mathcal{A}_{t}(+1,+1)\right|+\left|\mathcal{A}_{t}(-1,-1)\right|+2\left|\mathcal{A}_{t}(+1,-1)\right| \\
\left.=\left|\Gamma_{n}^{0}\right|^{-1} n^{-1} \sum_{t=1}^{n} \sum_{\left\{e_{1}, e_{2}\right\}}\left|\mathcal{A}_{t}\left(e_{1}, e_{2}\right)\right| \quad \text { by }(3.11)\right) \\
=\left|\Gamma_{n}^{0}\right|^{-1} n^{-1} \times \sum_{t=1}^{n}\left|\left\{(V, W): H_{V, W}(t)=m\right\}\right| \\
=\mathbb{P}\left(H_{V, W}\left(U_{n}\right)=m\right) .
\end{aligned}
$$




\section{Proof of Theorem 4.1}

In this section we prove that the size of the incomparability window of a typical element for a random poset with one factor converges to a Brownian excursion at a uniformly random time under the correct scaling.

Theorem 4.1 If $P$ is chosen uniformly at random from $\mathcal{P}_{0}$ and $\mathcal{X}$ is uniform over the ground set $[n]$ independent of $P$, then

$$
\frac{I_{P}(\mathcal{X})}{\sqrt{n}} \stackrel{D}{\Rightarrow} \frac{1}{\sqrt{2}} B^{\mathrm{ex}}(U)
$$

where $B^{\mathrm{ex}}$ is standard Brownian Excursion on $[0,1]$ and $U \sim$ Uniform $[0,1]$ independent of $B^{\mathrm{ex}}$.

Recall that by Lemma 2.2, this implies the main result.

4.1 The Distribution of $\sigma_{0}$. Instead of working directly with random posets we will work with the corresponding random walks as described in Section 3 and hence we begin with a convenient description of the uniform measure on $\Gamma_{n}^{0}$. Recall that by Lemma 3.7, $\sigma_{0}$ is the uniform measure on $\Gamma_{n}^{0}$. Let $Z^{(1)}$ and $Z^{(2)}$ be two independent random walks starting at the origin with

$$
Z_{k}^{(i)}=\sum_{l=1}^{k} z_{l}^{(i)},
$$

where the $z_{l}^{(i)}$ are i.i.d. \pm 1 with equal probability for $i=1,2$. Let $(V, W)$ be chosen uniformly at random from $\Gamma_{n}^{0}$. Let $X \stackrel{D}{=} Y \mid Z$ denote that the random variable $X$ is distributed like $Y$ conditioned on the event $Z$. By definition,

$$
(V, W) \stackrel{D}{=}\left(Z^{(1)}, Z^{(2)}\right) \mid Z_{k}^{(1)}>Z_{k}^{(2)} \text { for } 0<k<n, Z_{n}^{(1)}=Z_{n}^{(2)} .
$$

Consider the random walks $Z^{(1)}+Z^{(2)}$ and $Z^{(1)}-Z^{(2)}$. Note that unlike $Z^{(1)}$ and $Z^{(2)}$, these random walks are dependent. Their joint distribution can be described in the following way:

Let $\left\{\xi_{i}\right\},\left\{\xi_{i}^{\prime}\right\}$ for $1 \leq i \leq n$ be i.i.d. random variables taking values \pm 1 with probability $1 / 2$. Let $u_{i}$ be i.i.d. $\{0,1\}$ independent of $\left\{\xi_{i}\right\},\left\{\xi_{i}^{\prime}\right\}$. If we think of the variable $u_{i}$ being 1 if $z_{i}^{(1)}=z_{i}^{(2)}$ and 0 otherwise then it can be seen that $\frac{1}{2}\left(z_{i}^{(1)}+z_{i}^{(2)}\right)$ and $\frac{1}{2}\left(z_{i}^{(1)}-z_{i}^{(2)}\right)$ are distributed according to $\xi_{i} u_{i}$ and $\xi_{i}^{\prime}\left(1-u_{i}\right)$ respectively. For $1 \leq k \leq n$, let

$$
\begin{aligned}
Y_{k} & :=\xi_{1} u_{1}+\xi_{2} u_{2}+\ldots+\xi_{k} u_{k} \\
Y_{k}^{\prime} & :=\xi_{1}^{\prime}\left(1-u_{1}\right)+\xi_{2}^{\prime}\left(1-u_{2}\right)+\ldots+\xi_{k}^{\prime}\left(1-u_{k}\right) .
\end{aligned}
$$

Then,

$$
\left(\frac{1}{2}\left(Z^{(1)}+Z^{(2)}\right), \frac{1}{2}\left(Z^{(1)}-Z^{(2)}\right)\right) \stackrel{D}{=}\left(Y, Y^{\prime}\right) .
$$

Thus, we have the following distributional identity. 
Lemma 4.2 Let $(V, W)$ be uniform on $\Gamma_{n}^{0}$ and let $Y, Y^{\prime}$ be as above. Then

$$
\left(\frac{1}{2}(V+W), \frac{1}{2}(V-W)\right) \stackrel{D}{=}\left(Y, Y^{\prime}\right) \mid Y_{k}^{\prime}>0 \text { for } 0<k<n, Y_{n}^{\prime}=0 .
$$

Proof.

$$
\begin{aligned}
\left(\frac{1}{2}(V+W), \frac{1}{2}(V-W)\right) & \stackrel{D}{=}\left(\frac{1}{2}\left(Z^{(1)}+Z^{(2)}\right), \frac{1}{2}\left(Z^{(1)}-Z^{(2)}\right)\right) \mid Z \\
& \underline{D}\left(Y, Y^{\prime}\right) \mid Y_{k}^{\prime}>0 \text { for } 0<k<n, Y_{n}^{\prime}=0
\end{aligned}
$$

where above $Z$ is the event

$$
\left\{Z_{k}^{(1)}-Z_{k}^{(2)}>0 \text { for } 0<k<n, Z_{n}^{(1)}-Z_{n}^{(2)}=0\right\} .
$$

As an immediate corollary we obtain the distribution of the second marginal:

Corollary 4.3 Let $Y^{\prime}=x_{1}+x_{2}+\ldots+x_{k}$ where $\left\{x_{i}\right\}_{1 \leq i \leq n}$ are i.i.d. with $\mathbb{P}\left(x_{1}=0\right)=$ $1 / 2, \mathbb{P}\left(x_{1}=+1\right)=\mathbb{P}\left(x_{1}=-1\right)=1 / 4$. Let $(V, W)$ chosen uniformly from $\Gamma_{n}^{0}$. Then

$$
\frac{1}{2}(V-W) \stackrel{D}{=} Y^{\prime} \mid Y_{k}^{\prime}>0 \text { for } 0<k<n, Y_{n}^{\prime}=0 .
$$

4.2 An Upper Bound on the incomparability Window $I_{P}(\mathcal{X})$. Corollary 4.3 allows us to find the limiting distribution of the "average" incomparability window. For a poset $P \in \mathcal{P}_{0}$, define

$$
\hat{I}(P)=\frac{1}{n} \sum_{x \in[n]} I_{P}(x)
$$

Theorem 4.4 If $P$ is chosen uniformly at random from $\mathcal{P}_{0}$, then

$$
\frac{\hat{I}(P)}{\sqrt{n}} \stackrel{D}{\Rightarrow} \sqrt{2} \int_{0}^{1} B^{\mathrm{ex}}(t) d t
$$

where $B^{\operatorname{ex}}(\cdot)$ is a standard Brownian excursion on $[0,1]$.

Proof. By Corollary 3.16, $\hat{I}(P)=n^{-1} A(T)$ where for any two chain cover $T$ of $P, A(T)$ denotes the area between the piecewise-linear interpolations of the corresponding walks $V$ and $W$ over the time interval $[0, n]$. By abuse of notation, denote the pair of piecewise linear interpolated curves by $V_{s}, W_{s}$ for $s \in[0, n]$. Then

$$
\frac{1}{n} A(T)=\frac{1}{n} \int_{0}^{n}\left(V_{s}-W_{s}\right) d s=\sqrt{n} \int_{0}^{1} \frac{1}{\sqrt{n}}\left(V_{n t}-W_{n t}\right) d t .
$$

Now we apply Corollary 4.3. Let $\sigma^{2}:=\mathbb{E} x_{i}^{2}=1 / 2$. From the invariance principle for random walks excursions ( see [18] for example),

$$
\left(\frac{1}{\sigma \sqrt{n}} Y_{n t}^{\prime}, 0 \leq t \leq 1 \mid Y_{k}^{\prime}>0 \text { for } 0<k<n, Y_{n}^{\prime}=0\right) \stackrel{D}{\Rightarrow}\left(B^{\operatorname{ex}}(t), 0 \leq t \leq 1\right),
$$


where $Y_{n t}^{\prime}$ is the piecewise linear interpolation of $Y^{\prime}$. Here the weak convergence takes place in $C[0,1]$ equipped with uniform topology. It follows from the continuous mapping theorem that

The lemma now follows.

$$
\int_{0}^{1} 2 \frac{1}{\sqrt{n}} Y_{n t}^{\prime} d t \stackrel{D}{\Rightarrow} \int_{0}^{1} \sqrt{2} B^{e x}(t) d t
$$

Corollary 4.5 Let $P \in \mathcal{P}_{0}$ be uniformly distributed and let $\mathcal{X}$ be chosen uniformly from the ground set $[n]$ independent from $P$. Then

$$
\mathbb{P}\left(I_{P}(\mathcal{X})>n^{2 / 3}\right) \rightarrow 0
$$

Proof. From Theorem 4.4 it follows that $\mathbb{P}\left(\hat{I}(P) \leq n^{1 / 2+1 / 12}\right) \rightarrow 1$ as $n \rightarrow \infty$. Markov's inequality implies that $\mathbb{P}\left(I_{P}(\mathcal{X})>n^{2 / 3} \mid \hat{I}(P) \leq n^{1 / 2+1 / 12}\right) \rightarrow 0$ as $n \rightarrow \infty$ and the proof follows.

4.3 Conclusion of the Proof of Theorem 4.1. In this section we show that for a randomly chosen poset $P \in \mathcal{P}_{0}$, and for $\mathcal{X}$ a uniformly random element of $[n]$,

$$
\operatorname{Err}(P, \mathcal{X}):=\left|V\left(\tau_{P}(\mathcal{X})+I_{P}(\mathcal{X})\right)-V\left(\tau_{P}(\mathcal{X})\right)\right|+\left|W\left(\tau_{P}(\mathcal{X})+I_{P}(\mathcal{X})\right)-W\left(\tau_{P}(\mathcal{X})\right)\right|
$$

behaves as $o(\sqrt{n})$ in probability. We show that for typical elements of the poset, the fluctuations in the corresponding random walk representation are small.

Let $Y_{k}$ and $Y_{k}^{\prime}$ be the random walks as defined in (4.4). The next result shows that the probability that either $Y_{k}$ or $Y_{k}^{\prime}$ exhibits a deviation of more than $n^{1 / 3+\delta}$ in $n^{2 / 3}$ steps of the walks goes to 0 . Further, the probabilities go to zero even when conditioned on the event $\left\{Y_{k}^{\prime}>0 \forall 1 \leq k \leq n-1, Y_{n}^{\prime}=0\right\}$. Together with (4.7) the result will imply that the walks $V, W$ have fluctuations bounded by $n^{1 / 3+\delta}$ if we look at most $n^{2 / 3}$ steps away, with high probability. We make use of the following result for a simple symmetric random walk (SSRW).

Proposition 4.6 ([15], Lemma 2, page 78) For every $k \in \mathbb{N}$,

$$
\mathbb{P}(\text { The first return to } 0 \text { of a SSRW is at } 2 k)=\frac{1}{2 k-1}\left(\begin{array}{c}
2 k \\
k
\end{array}\right) 2^{-2 k}=\Omega\left(\frac{1}{k^{3 / 2}}\right) \text {. }
$$

Lemma 4.7 Let $Y, Y^{\prime}$ be as in (4.4) and let $G=\left\{Y_{k}^{\prime}>0 \forall 1 \leq k \leq n-1, Y_{n}^{\prime}=0\right\}$. Then, for all $1 \leq k \leq n$ and for any $\delta>0$,

$$
\mathbb{P}\left(\max _{0 \leq s \leq n^{2 / 3}}\left|Y_{k+s}-Y_{k}\right|>n^{1 / 3+\delta} \mid G\right) \rightarrow 0
$$

and

$$
\mathbb{P}\left(\max _{0 \leq s \leq n^{2 / 3}}\left|Y_{k+s}^{\prime}-Y_{k}^{\prime}\right|>n^{1 / 3+\delta} \mid G\right) \rightarrow 0
$$

as $n \rightarrow \infty$. 
Proof. For any $1 \leq k \leq n$, we can bound the probability of the unconditional events above by the Chernoff bound as follows

$$
\begin{aligned}
\mathbb{P}\left(\max _{0 \leq s \leq n^{2 / 3}}\left|Y_{k+s}-Y_{k}\right|>n^{1 / 3+\delta}\right) & \leq \sum_{0 \leq s \leq n^{2 / 3}} \mathbb{P}\left(\left|Y_{s}\right|>n^{1 / 3+\delta}\right) \\
& \leq 2 n^{2 / 3} e^{-n^{2 \delta / 3} / 4}
\end{aligned}
$$

Similarly,

$$
\mathbb{P}\left(\max _{0 \leq s \leq n^{2 / 3}}\left|Y_{k+s}^{\prime}-Y_{k}^{\prime}\right|>n^{1 / 3+\delta}\right) \leq 2 n^{2 / 3} e^{-n^{2 \delta / 3} / 4}
$$

Further, we have

$$
\begin{aligned}
& \mathbb{P}\left(\text { The first return to } 0 \text { of } Y_{k}^{\prime} \text { is at } n\right) \\
= & \left.\sum_{0 \leq i \leq n}^{*} \mathbb{P}\left(\mid\left\{j: u_{j}=1\right)\right\} \mid=i\right) \mathbb{P}(\text { the first return to } 0 \text { of a SSRW is at } n-i) \\
= & \left.\Omega\left(\frac{1}{n^{3 / 2}}\right) \sum_{0 \leq i \leq n}^{*} \mathbb{P}\left(\mid\left\{j: u_{j}=1\right)\right\} \mid=i\right) \quad \text { (by Proposition 4.6) }
\end{aligned}
$$

where the sum $\sum^{*}$ is restricted to those $i$ for which $n-i \in 2 \mathbb{Z}_{\geq}$, and therefore

$$
\mathbb{P}(G)=\Omega\left(\frac{1}{n^{3 / 2}}\right)
$$

By (4.17) and (4.19), we have

$$
\begin{aligned}
\mathbb{P}\left(\max _{0 \leq s \leq n^{2 / 3}}\left|Y_{k+s}-Y_{k}\right|>n^{1 / 3+\delta} \mid G\right) \leq & \frac{\mathbb{P}\left(\max _{0 \leq s \leq n^{2 / 3}}\left|Y_{k+s}-Y_{k}\right|>n^{1 / 3+\delta}\right)}{\mathbb{P}(G)} \\
& \leq O(1) \frac{n^{2 / 3} e^{-n^{2 \delta / 3} / 4}}{n^{-3 / 2}} \rightarrow 0 .
\end{aligned}
$$

A similar argument using (4.18) and (4.19) shows that

$$
\mathbb{P}\left(\max _{0 \leq s \leq n^{2 / 3}}\left|Y_{k+s}^{\prime}-Y_{k}^{\prime}\right|>n^{1 / 3+\delta} \mid G\right) \rightarrow 0
$$

Corollary 4.8 If $(V, W)$ are drawn uniformly from $\Gamma_{n}^{0}$, then for all $1 \leq k \leq n$ and $\delta>0$,

$$
\mathbb{P}\left(\max _{0 \leq s \leq n^{2 / 3}}|V(k+s)-V(k)|>2 n^{1 / 3+\delta}\right) \rightarrow 0
$$


and

$$
\mathbb{P}\left(\max _{0 \leq s \leq n^{2 / 3}}|W(k+s)-W(k)|>2 n^{1 / 3+\delta}\right) \rightarrow 0
$$

Proof. The claim follows by adding the bounds from (4.15) and (4.16) from Lemma 4.7 and the description of $(V, W)$ in (4.7).

We can now complete the proof of Theorem 4.1.

Proof of Theorem 4.1. Recall that we would like to show that

$$
\frac{1}{\sqrt{n}} I_{P}(\mathcal{X}) \stackrel{D}{\Rightarrow} \frac{1}{\sqrt{2}} B^{e x}(U)
$$

Let $(P,(V, W))$ be chosen according to 2-dimensional marginal of $\omega$ on $\mathcal{P}_{0} \times \Gamma_{n}^{0}$ and let $\mathcal{X}$ be chosen uniformly at random from $[n]$ independent of $(P,(V, W))$. By Corollary 4.5 , with probability going to $1, I_{P}(\mathcal{X}) \leq n^{2 / 3}$. Therefore, by part $(2)$ of Theorem 3.5 , with high probability

$$
\begin{aligned}
\left|I_{P}(\mathcal{X})-H_{V, W}\left(\tau_{P}(\mathcal{X})\right)\right| & \leq \max _{0 \leq k \leq n^{2 / 3}}\left|V\left(\tau_{P}(\mathcal{X})+k\right)-V\left(\tau_{P}(\mathcal{X})\right)\right| \\
& +\max _{0 \leq k \leq n^{2 / 3}}\left|W\left(\tau_{P}(\mathcal{X})+k\right)-W\left(\tau_{P}(\mathcal{X})\right)\right| .
\end{aligned}
$$

By Corollary 4.8,

$$
\frac{1}{\sqrt{n}}\left(\max _{0 \leq k \leq n^{2 / 3}}|V(\tau(\mathcal{X})+k)-V(\tau(\mathcal{X}))|+\max _{0 \leq k \leq n^{2 / 3}}|W(\tau(\mathcal{X})+k)-W(\tau(\mathcal{X}))|\right) \stackrel{P}{\rightarrow} 0
$$

where $\stackrel{P}{\rightarrow}$ denotes convergence in probability. Therefore,

$$
\frac{1}{\sqrt{n}}\left|I_{P}(\mathcal{X})-H_{V, W}\left(\tau_{P}(\mathcal{X})\right)\right| \stackrel{P}{\rightarrow} 0
$$

By Lemma 3.9, $H_{V, W}\left(\tau_{P}(\mathcal{X})\right)$ equals $H_{V, W}\left(\tau_{V, W}(\mathcal{C})\right)$ where $\mathcal{C}$ is some uniform random variable on $[n]$ which is independent of $(V, W)$. Hence,

$$
\frac{1}{\sqrt{n}}\left|I_{P}(\mathcal{X})-H_{V, W}\left(\tau_{V, W}(\mathcal{C})\right)\right| \stackrel{P}{\rightarrow} 0
$$

Let $(V, W)$ be chosen according to $\sigma_{0}$. The invariance principle for random walk excursions implies (see 4.11) that

$$
\frac{1}{\sqrt{n}} H_{V, W}\left(U_{n}\right) \stackrel{D}{\Rightarrow} \frac{1}{\sqrt{2}} B^{\mathrm{ex}}(U) .
$$

By Theorem 3.10, $H_{V, W}\left(\tau_{V, W}(\mathcal{C})\right)$ has same distribution as $H_{V, W}\left(U_{n}\right)$ and therefore

$$
\frac{1}{\sqrt{n}} H_{V, W}\left(\tau_{V, W}(\mathcal{C})\right) \stackrel{D}{\Rightarrow} \frac{1}{\sqrt{2}} B^{\operatorname{ex}}(U) .
$$

This implies the claim

$$
\frac{1}{\sqrt{n}} I_{P}(\mathcal{X}) \stackrel{D}{\Rightarrow} \frac{1}{\sqrt{2}} B^{\mathrm{ex}}(U)
$$




\section{Distribution of Height}

In this section, we prove Theorem 1.2 showing that the height of random width- 2 poset is $n / 2$ with a Gaussian fluctuation.

Proof of Theorem 1.2. Let $M_{1}<M_{2}<\ldots<M_{k}$ be the factors of $P$ with $T_{i}=\left(L_{i}, R_{i},<\right)$ being a two chain cover of $M_{i}$. The longest chain in $P$ can be constructed by concatenating the longer of the two chains from each of the $k$ factors. Thus the height of $P$ is given by

$$
h(P)=\sum_{i=1}^{k} \max \left(\left|L_{i}\right|,\left|R_{i}\right|\right) .
$$

As in Lemma 2.3, it is easy to check that that the height is essentially determined by the largest factor which is of size at least $n-O(\log n)$. We may proceed analogously to the proof of Theorem 1.1 to conclude that it is enough to prove the above theorem for the special case when the poset $P$ is chosen uniformly at random from $\mathcal{P}_{0}$, i.e. it has only one factor.

For $P \in \mathcal{P}_{0}$, let $(V, W) \in \phi(P)$ be an associated pair of walks. It is easy to see that

$$
h(P)=\frac{n+|V(n)|}{2}=\frac{n+|W(n)|}{2} .
$$

Thus, we would like to find the limiting distribution of $(V(n)+W(n)) / 4 \sqrt{n}$. The next lemma completes the proof once we recall the distributional identity given in Lemma 4.2.

Lemma 5.1 Let $Y, Y^{\prime}$ be as in the paragraph preceding Lemma 4.2. Then

$$
\frac{2 Y_{n}}{\sqrt{n}} \mid Y_{k}^{\prime}>0 \text { for } 0<k<n, Y_{n}^{\prime}=0 \stackrel{D}{\Rightarrow} N(0,1)
$$

as $n \rightarrow \infty$.

Proof. Let $\Phi(\cdot)$ denote the standard normal distribution function. Let $G$ denote the event $\left\{Y_{k}^{\prime}>0\right.$ for $\left.0<k<n, Y_{n}^{\prime}=0\right\}$. Fix $x \in \mathbb{R}$. We then have

$$
\begin{aligned}
\mathbb{P}\left(\frac{2 Y_{n}}{\sqrt{n}} \leq x \mid G\right)-\Phi(x) & =\sum_{0 \leq m \leq n}^{*}\left(\mathbb{P}\left(\frac{2 Y_{n}}{\sqrt{n}} \leq x \mid G, \sum_{i=1}^{n} u_{i}=m\right)-\Phi(x)\right) \mathbb{P}\left(\sum_{i=1}^{n} u_{i}=m \mid G\right) \\
& =\sum_{0 \leq m \leq n}^{*}\left(\mathbb{P}\left(\frac{2}{\sqrt{n}} \sum_{i=1}^{m} \xi_{i} \leq x\right)-\Phi(x)\right) \mathbb{P}\left(\sum_{i=1}^{n} u_{i}=m \mid G\right)
\end{aligned}
$$

where the sum $\sum^{*}$ always includes the added restriction $n-m \in 2 \mathbb{Z}$. The lemma now follows from a simple application of the Berry-Esséen bound (see for example [13]) once we prove that $\sum_{i=1}^{n} u_{i}$ is concentrated around $n / 2$ even when conditioned on $G$. More precisely, we want to show,

$$
\mathbb{P}\left(\left|\sum_{i=1}^{n} u_{i}-n / 2\right|>n^{3 / 4} \mid G\right) \rightarrow 0 .
$$


Note that

$$
\mathbb{P}\left(\left|\sum_{i=1}^{n} u_{i}-n / 2\right|>n^{3 / 4} \mid G\right) \leq \frac{\mathbb{P}\left(\left|\sum_{i=1}^{n} u_{i}-n / 2\right|>n^{3 / 4}\right)}{\mathbb{P}(G)} \leq \frac{c_{1} \exp \left(-c_{2} n^{1 / 2}\right)}{\mathbb{P}(G)},
$$

for suitable constants $c_{1}, c_{2}>0$. By (4.19)

$$
\mathbb{P}(G)=\Omega\left(\frac{1}{n^{3 / 2}}\right)
$$

and the claim follows.

\section{Conclusions}

The results in this paper should be viewed as a first step in the analysis of random posets of bounded width. Our results provide the asymptotic distribution of the number of elements incomparable to a random element. However, more detailed information is desirable.

In our results we find a distribution $F$ such that for a random element $x$, there is a maximal chain of incomparable elements $y_{i(0)}<\ldots<y_{j(0)}$ and $j(0)-i(0)$ is distributed according to $F$. In fact one would expect to extract more detailed information about the "neighborhood" of $x$ and that it has the following structure: $x=x_{0}$ belongs to a chain $<$ $\cdots<x_{-1}<x_{0}<x_{1}<\cdots$ and there exists another chain $\cdots<y_{-1}<y_{0}<y_{1}<\cdots$ such that each element $x_{k}$ of the first chain is incomparable to elements $y_{i(k)}<\cdots<y_{j(k)}$ of the other chain. Then it would be desirable to identify the (joint) distribution of $i(k+1)-i(k)$ and $j(k+1)-j(k)$ for small values of $k$. Even more detailed information is desirable in terms of the joint distribution of the $x$ 's, the $y$ 's and the elements incomparable to $y$ 's etc. As mentioned in the introduction, it would also be desirable to extend the analysis here to set of bounded width greater than 2 .

\section{Acknowledgments}

E.M. is grateful to Graham Brightwell for discussions which initiated this work. The authors are grateful to David Aldous and Steve Evans for pointing out the distribution of the height of a Brownian excursion.

\section{REFERENCES}

[1] D. Aldous. The continuum random tree III, The Annals of Prob. 21(1), 248-289, 1993.

[2] J. Baik, P. Deift and K. Johansson. On the distribution of the length of the longest increasing subsequence of random permutations, J. Amer. Math. Soc. 12, 1119-1178, 1999.

[3] P. Billingsley. Probability and Measure, Third edition, Wiley Series in Probability and Mathematical Statistics, A Wiley-Interscience Publication. John Wiley \& Sons, Inc., New York, 1995.

[4] B. Bollobás. Modern Graph Theory, Springer-Verlag, Berlin, 1998.

[5] B. Bollobás. Random Graphs, Academic Press, London, 1985. 
[6] B. Bollobás and P. Winkler. The longest chain among random points in Euclidean space, Proc. Amer. Math. Soc. 103(2), 347-353, 1988.

[7] B. Bollobás, G. Brightwell. Box-spaces and random partial orders, Trans. Amer. Math. Soc. 324, 59-72, 1991.

[8] B. Bollobás, G. Brightwell. The height of a random partial order: concentration of measure, Ann. of Appl. Prob., 2, 1009-1018, 1992.

[9] G. Brightwell. Models of random partial orders, Surveys in Combinatorics, London Math. Soc. Lecture Notes Series, ed. Keith, 187, 53-83, 1993.

[10] G. Brightwell and S. Goodall. The number of partial orders of fixed width, Order, 13, 315-337, 1996.

[11] C. Daskalakis, R. Karp, E. Mossel, S. Riesenfeld, E. Verbin. Sorting and Selection in Posets, preprint, 2007, available at http://arxiv.org/abs/0707.1532.

[12] D. Dhar. Entropy and phase transitions in partially ordered sets, J. Math. Phys., 19(8), 1711-1713, 1978.

[13] R. Durrett. Probability: Theory and Examples, Duxbury Press, 1996.

[14] U. Faigle and Gy. Turán. Sorting and Recognition Problems for Ordered Sets, SIAM J. Comput. 17(1), 100-113, 1988.

[15] W. Feller. An Introduction to Probability Theory and its Applications. Vol. I. John Wiley \& Sons, Inc., New York-London-Sydney 1968.

[16] K. Itô, H.P. McKean. Diffusion Processes and their Sample Paths, Springer, 1965.

[17] S. Janson, T. Luczak, A. Rucinski. Random Graphs, Wiley, 2000.

[18] W. D. Kaigh. An invariance principle for random walk conditioned by a late return to zero. Ann. Prob., 4(1), 115-121, 1976.

[19] D. L. Kleitman and B.R. Rothschild. Asymptotic enumeration of partial orders on a finite set, Trans. Amer. Math. Soc., 205, 205-220, 1975.

[20] D. L. Kleitman and B.R. Rothschild. A phase transition on partial orders, Phys. A 96, 254-259, 1979.

[21] H.J. Prömel and, A. Steger and A. Taraz. Phase transitions in the evolution of partial orders, Jour. Comb. Theory Ser. A, 94(2), 230-275, 2001.

[22] K. Strimmer and V. Moulton. Likelihood analysis of phylogenetic networks Using directed graphical models, Mol. Biol. and Evol., 17(6) 875-881, 2000.

[23] K. Strimmer, C. Wiuf and V. Moulton. Recombination analysis using directed graphical models, Mol. Biol. and Evol., 17(6) 875-881, 2000.

[24] P. Winkler, Random orders, ORDER 1, 317-331, 1985. 